\section{Short telomeres, damaged hearts}

ovement.

Different

brain areas were activated depending on whether the crows were

$\sum$ exposed to an innate threat (a taxidermy hawk), a known human threat (the person who had initially captured them) or a potential threat

(an unknown person holding a dead crow). The researchers suggest that mobbing behaviour could be guided by distinct neural circuits involved in innate responses, memory and learning.

Proc. R. Soc. B 280, 20131046 (2013)

\section{QUANTUM COMMUNICATION}

\section{Broken quantum links still work}

In quantum physics, linked photons can provide ultrasecure communication even if the link between them is lost.

Quantum communication, which physicists hope can thwart eavesdropping attempts, often relies on entanglement — tight links between the quantum states of two particles. However, that link is easily broken by background noise, making schemes difficult to implement in real-world situations. Zheshen Zhang and his team at the Massachusetts Institute of Technology in Cambridge showed that correlations between the light pulses exchanged by two parties over an optical fibre are strong enough to convey messages even if noise destroys the photons' entanglement.

This is the first experimental demonstration of a communication scheme with broken entanglement. The work also supports the idea that technologies using entanglement could be made to work even in practical situations.

Phys. Rev. Lett. 111, 010501

(2013)
Chromosome tips might explain why mice carrying mutations for a heritable form of muscular dystrophy do not display the heart problems that eventually kill people with the disease.

Humans with Duchenne muscular dystrophy die young from cardiorespiratory failure, but mice with similar genetic mutations have normal lifespans and only mild symptoms. However, researchers led by Helen Blau at Stanford University in California, showed that mice with the mutation did display severe cardiac defects if, like humans, they also had shortened telomeres, the protective caps on the ends of chromosomes. Heart muscle in these mice showed signs of oxidative stress, a kind of chemical damage associated with shorter telomeres, and this damage could be ameliorated with antioxidants. Follow-up work on heart muscle tissue from four people who had Duchenne muscular dystrophy showed that all four had very short telomeres.

The results could be used to improve animal models for Duchenne muscular dystrophy and to develop ways authors say.

Nature Cell Biol. http://dx.doi. org/10.1038/ncb2790 (2013)

\section{NANOTECHNOLOGY}

\section{Single-molecule electric switch}

Ultraviolet light alters the conductance of organic molecules deposited on graphene, and could be used to manipulate devices that operate on molecular scales.

Molecular electronics hold promise for making computer chips smaller, but researchers have struggled to control the electrical behaviour of individual molecules. To address this problem, Xuefeng to slow heart damage, the

COMMUNITY CHOICE

The most viewed papers in science

\title{
BIOELECTRONICS
}

\section{An ear by printing}

\section{จ HIGHLY READ \\ on www.acs.org in June}

A special printer loaded with silver nanoparticles, silicone and living cells can print a three-dimensional bionic ear with functional electronics.

Michael McAlpine at Princeton University in New Jersey and his colleagues used a computer to design a humansized 'ear' with a spiral antenna and electrodes shaped like the cochlea of the inner ear. The printer made the device by building up all the materials layer by layer, encasing the electronics in a hydrogel scaffold seeded with specialized cells. The structure was placed in a nutrient broth to grow the cells into cartilage.

Although the bionic ears do not detect sound waves, they can receive radio signals at frequencies within and beyond the normal range of hearing through the cartilage-covered antenna. The work shows that wet, squishy biological materials can be interwoven with functioning electronics even in complex structures, the authors say. Nano Lett. 13, 2634-2639 (2013)

Guo and Zhongfan Liu at Peking University in Beijing and their colleagues used derivatives of diarylethene molecules, which change shape when exposed to light. This changes how electrons pass through the molecules and so alters conductivity. These 'single-molecule junctions' functioned reproducibly as electrical switches.

Angew. Chem. Int. Edn.

http://dx.doi.org/10.1002/

anie.201304301 (2013)

\section{HOMEOSTASIS}

\section{Fat cells that} sense cold

Certain fat cells can switch on heat-generating pathways directly, without being prompted by the nervous system.

Known for its ability to convert chemical energy to heat, brown fat warms up when cold-sensing neural circuits release the neurotransmitter noradrenaline. Without $\beta$-adrenergic receptors to

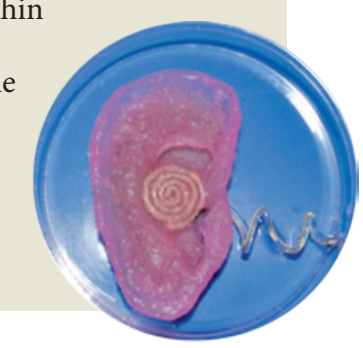

sense these

signals, the response of brown fat to the cold is limited.

A team led by Bruce Spiegelman at the DanaFarber Cancer Institute in Boston, Massachusetts, found that other types of fat cell can activate genes to boost heat production in chilly environments - even if they lack $\beta$-adrenergic receptors. When exposed to temperatures $33^{\circ} \mathrm{C}$ or below, these white and beige fat cells sharply increased their expression of two thermogenesis genes within a matter of hours.

A large part of coldinduced heat production from fat could come from subcutaneous fat tissue that senses temperature directly, the authors say.

Proc. Natl Acad. Sci. USA

http://dx.doi.org/10.1073/ pnas.1310261110 (2013)

\section{ONATURE.COM}

For the latest research published by Naturevisit:

www.nature.com/latestresearch 